\title{
JUIN 1958
}

\section{REVUE}

\section{INTERNATIONA LE}

\author{
DE LA
}

\section{CROIX-ROUGE}

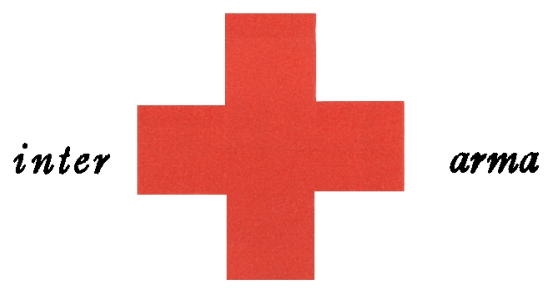

caritas

COMITE INTERNATIONAL DE LA CROIX-ROUGE GENEVE 


\section{COMITÉ INTERNATIONAL DE LA CROIX-ROUGE à Genève}

President d'honnewr: M. MAX HUBER, docteur en droit, ancien président de la Cour permanente de justice internationale

MM. LEOPOLD BOISSIER, docteur en droit, professeur honoraire de l'Université de Genève, ancien secrétaire général de l'Union interparlementaire, président (1946) 1

JACQUES CHENEVIERE, docteur ès lettres h. c. (1919)

M110 LUCIE ODIER, ex-chef du Service des infirmières-visiteuses de la Section genevoise de la CroixRouge suisse (1930),

MM. CARL J. BURCKHARDT, docteur en philosophie, ancien ministre de Suisse en France (1933)

MARTIN BODMER, docteur en philosophie h. c., vice-président (1940)

ERNEST GLOOR, docteur en médecine (1945)

PAUL CARRY, docteur en droit, professeur à l'Université de Genève (1946)

PAUL RUEGGER, ancien ministre de Suisse en Italie et en Grande-Bretagne, membre de la Cour Permanente d'Arbitrage (1948)

HENRI GUISAN, général, ancien commandant en chef de l'armée suisse (1948)

ALFREDO VANNOTTI, docteur en médecine, professeur à I'Université de Lausanne (1049)

RODOLFO OLGIATI, ancien directeur du Don suisse (1949)

Mile MARGUERITE VAN BERCHEM, ancien chef de service de l'Agence centrale des prisonniers de guerte (1951)

MM .FRÉEÉRIC SIORDET, avocat, conseiller du Comité international de la Croix-Rouge de 1943 à 1951, vice-président (1951)

MARCEL JUNOD, docteur en médecine, délégué du Comité international de la Croix-Rouge de 1035 à 1946 (1952)

GUILLAUME BORDIER, ingénieur dipl. E.P.F., M.B.A. Harvard, banquier (1955)

\section{Direction :}

MM. ROGER GALLOPIN, docteur en droit, directeur exécutif

JEAN S. PICTET, docteur en droit, đirecteur des Affaires générales

EUOUARD de BONDELI, sous-directeur, Services financiers et administratifs

CLAUDE PILLOUD, sous-directeur, Service juridique 


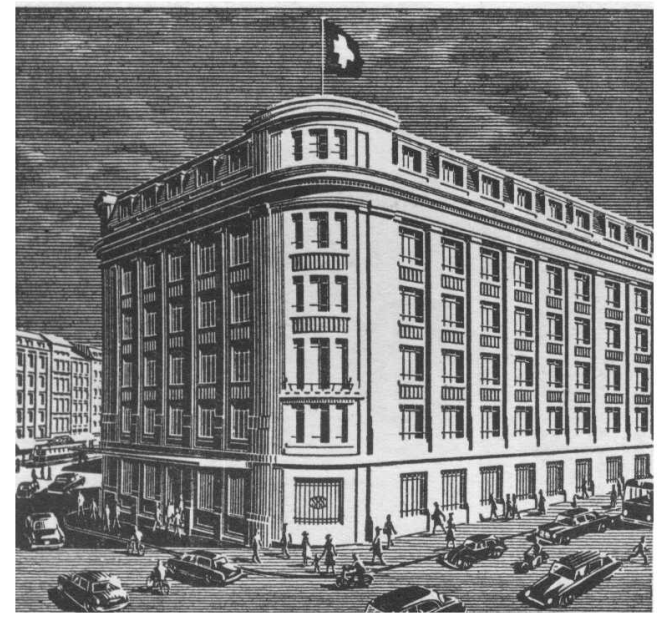

SOCIETEE DE

BANQUE SUISSE SCHWEIZERISCHER BANKVEREIN SOCIETÀ DI BANCA SVIZZERA SWISS BANK CORPORATION

\section{GENÈVE}

BALE BIENNE LA OHAUX-DE-FONDS LAUSANNE NEUOHATEL ST-GALL SOHAFFHOUSE ZURICH LONDRES NEW-YORK

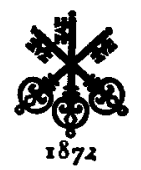

CAPITAL ET RESERVES FRS 280 MILLIONS 

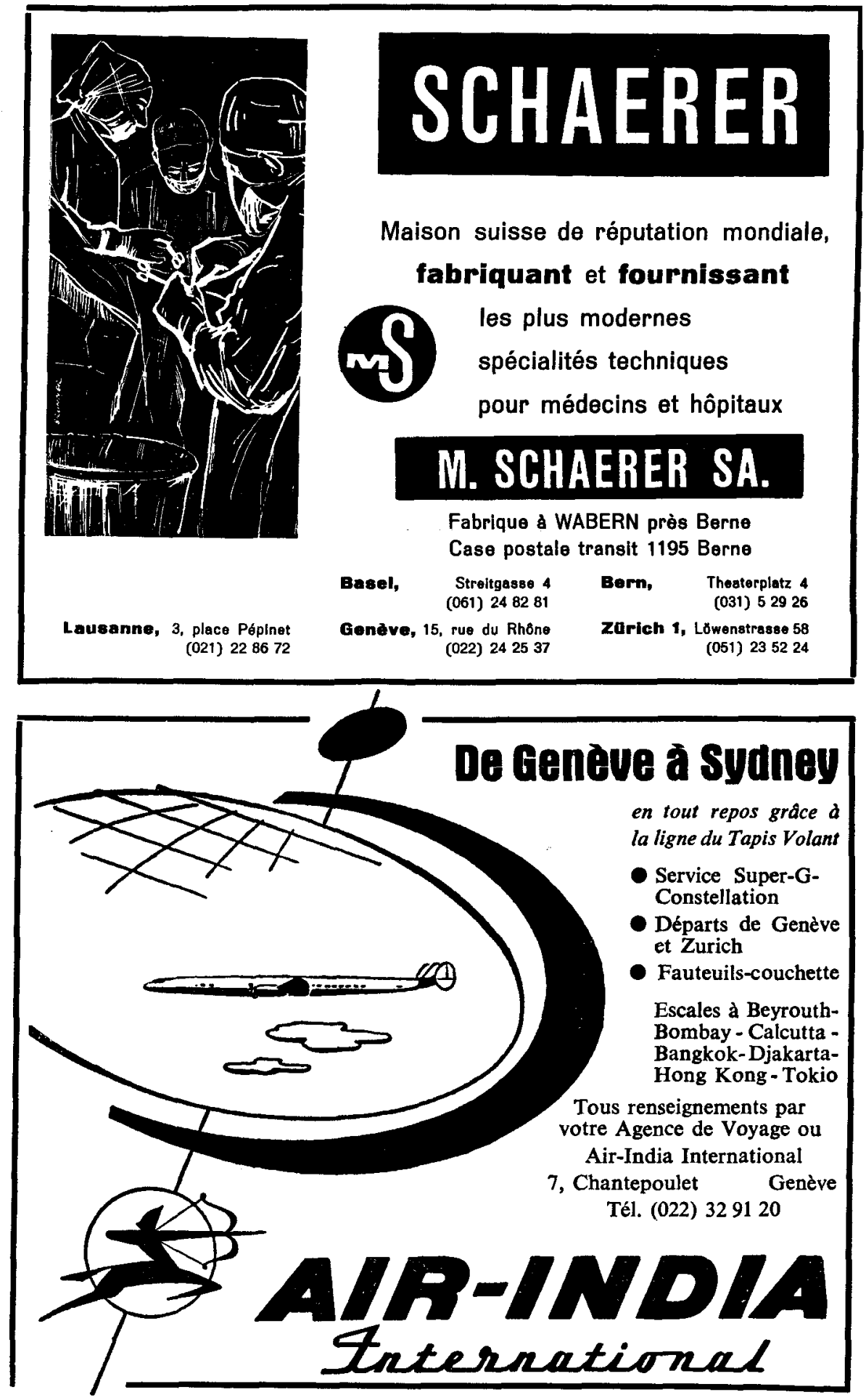


\title{
REVUE INTERNATIONALE \\ DE LA CROIX-ROUGE
}

\author{
QUARANTIËME ANNEEE - N०474 \\ JUIN 1958
}

SOMMAIRE

Pages

M. Inoué : Préparation nationale de la Croix-Rouge devant les dangers d'une guerre atomique . . . . . . . . . . . . . 295

COMITÉ INTERNATIONAL DE LA CROIX-ROUGE Mission du CICR en Tchécoslovaquie et en Allemagne orientale 306 Pour faire connaître le CICR et son cuvre . . . . . . . . 309

\section{CROIX-ROUGE INTERNATIONALE}

L'inauguration du Pavillon de la Croix-Rouge internationale ’̀ l'Exposition universelle de Bruxelles

\section{NOUVELLES DE SOCIÉTÉS NATIONALES}

Colombie

Indonésit.

Italie

Pays-Bas

Suisse

\section{CHRONIQUE}

L'école et les Conventions de Genève . . . . . . . . . . . 329

En souvenir d'Elsa Brandström (J.Z.) . . . . . . . . . . 332

\section{FAITS ET DOCUMENTS}

La $I I I^{\mathrm{e}}$ Conférence internationale de Protection civile (J.-P. S.) $33^{8}$ La Croix-Rouge et l'assistance aux détenus politiques (H. C.) $34^{0}$ La santé du monde: Hier et demain . . . . . . . . . . . 342

\section{BIBLIOGRAPHIE}

A TRAVERS LES REVUES 


\title{
SUPPLÉMENTS DE LA REVUE
}

\author{
EN LANGUE ANGLAISE
}

C. Pilloud : Reservations to the I949 Geneva Conventions (I). - A mission of the ICRC in Czechoslovakia and East Germany. - Red Cross activities in China.

EN LANGUE ESPAGNOLE

C. Pilloud : Las reservas a los Convenios de Ginebra de 1949 (I). - Misión del CICR en Checoeslovaquia y en Alemania Oriental. - La actividad de la Cruz Roja china.

\section{EN LANGUE ALLEMANDE}

C. Pilloud: Die Vorbehalte zu den Genfer Abkommen (I). - Mission des IKRK in der Tschechoslovakei und in Ostdeutschland. - Die Tätigkeit des Chinesischen Roten Kreuzes.

LA

REVUE INTERNATIONALE DE LA CROIX-ROUGE est publiée chaque mois par le Comité international de la Croix-Rouge

7, avenue de la Paix, Genève (Suisse) - Compte de chèques postaux I. 1767

Abonnement un an: Fr. 20,- ; le numéro Fr. 2,-

Pour les pays suivants : Algérie, Allemagne, Autriche, Belgique, Danemark, Finlande, France, Italie, Luxembourg, Maroc, Norvège, Pays-Bas, Portugal, Sarre. Suede, Tunisic. Vatican, on peut s'abonner aupres des bureaux de poste (Fr. s. 20,50).

Rédaction : JEAN-G. LOSSIER 